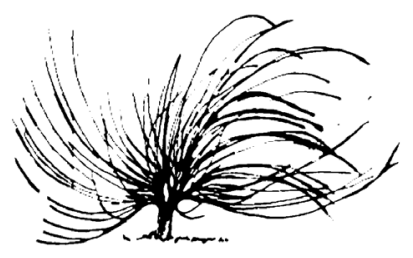

\title{
Relación entre la tasa de aprobación de estudiantes de la Facultad de Medicina y la evaluación del desempeño docente del periodo 2017
}

\author{
Fabiola Quiroz Condori ${ }^{l}$ \\ Universidad Federada de Costa Rica, San Judas Tadeo \\ Costa Rica \\ calidad@usanjudas.ac.cr \\ Leonardo Roque Pujol ${ }^{2}$ \\ Universidad Federada de Costa Rica, San Judas Tadeo \\ Costa Rica \\ leonardo@usanjudas.ac.cr \\ María de los Ángeles Echeverría Sáenz ${ }^{3}$ \\ Universidad Federada de Costa Rica, San Judas Tadeo \\ Costa Rica \\ draecheverria@usanjudas.ac.cr \\ Elizabeth Vargas Rojas ${ }^{4}$ \\ Universidad Federada de Costa Rica, San Judas Tadeo \\ Costa Rica \\ dravargas@usanjudas.ac.cr
}

\section{(c) (i) () $\Theta$}

Recibido: 24 de setiembre de 2018. Aprobado: 10 de abril de 2019.

http://dx.doi.org/10.15359/rep.14-1.11

1 Psicóloga, licenciada, Universidad Católica de Costa Rica.

2 Curriculista, máster, Universidad Latina de Costa Rica.

3 Fisióloga, doctora, Universidad Latina de Costa Rica.

4 Psicóloga, licenciada, Universidad Católica de Costa Rica. 


\title{
Resumen
}

Este artículo de investigación se ubica en el tipo de estudio científico que aborda la relación entre la tasa de aprobación de estudiantes y la evaluación del desempeño docente de la Facultad de Medicina de la Universidad Federada de Costa Rica, San Judas Tadeo. El estudio describe el tipo de relación, simétrica o asimétrica, entre la tasa de aprobación de estudiantes y la calificación que el alumnado le otorga a sus docentes. Esto se realiza desde un enfoque exploratorio, con el fin de establecer, a partir de los ítems evaluados, los elementos relacionados con el desempeño del profesorado en el aula que inciden en la evaluación de los aprendizajes estudiantiles y, por tanto, en su rendimiento académico. El estudio adopta una metodología cuantitativa con un alcance descriptivo. La información fue recogida por un período de tres cuatrimestres durante el año 2017. El proceso de investigación incorpora el análisis de competencias específicas que debe poseer el personal docente en el desarrollo del proceso de enseñanza. El resultado de la investigación determina que el cuerpo docente de la carrera de medicina posee altas competencias profesionales para ejercer su labor. Algunos aspectos de mejora encontrados están relacionados con determinadas competencias en el ámbito actitudinal.

Palabras clave: Evaluación, desempeño, docente, tasa, aprobación, estudiante.

\begin{abstract}
This article reports on the findings of a study that sought to address the relationship between student course evaluations and passing rates of students enrolled at Universidad Federada San Judas Tadeo, School of Medicine in Costa Rica. The study aimed to describe correlations between the different variables measured using an exploratory approach in order to evaluate certain aspects of teacher performance that may affect student learning and academic performance. The study described the type relationship, symmetric or asymmetric, between passing rates and the teacher evaluation performed by
\end{abstract}


the students, from an exploratory approach, in order to establish, from the measured items, the possible components related to teacher classroom performance that may affect student learning and, therefore, their academic performance. The study utilized a quantitative approach with a descriptive method. The information was collected during the three academic quarters in 2017. The research process incorporated the analysis of specific competencies that professors must possess in the development of the teaching process. This study found that teachers of medical sciences are highly competent in their field. There is room for improvement with regard to attitudinal competencies associated with teaching.

Keywords: Teacher, performance, evaluation, student, passing

\section{Introducción}

La calidad en todos los procesos académicos y administrativos es fundamental para el mejoramiento y el desarrollo de una cultura universitaria acorde con las exigencias educativas de la sociedad actual. El personal docente universitario juega un papel protagónico dentro de ese proceso, ya que es el responsable, junto con el estudiantado, de generar actividades de mediación para la construcción del conocimiento en función del desarrollo personal en tanto que dan respuestas a las necesidades sociales.

El personal docente universitario es profesional con conocimientos y habilidades para convertir la experiencia educativa en un fenómeno enriquecedor y participativo que posibilite la profundización y la creación de conocimiento. La profesionalización de la práctica docente en el campo de la salud conlleva a consolidar, de forma efectiva, las competencias para saber qué enseñar, cómo enseñar, a quiénes se enseña y para qué, con determinada finalidad. Es por ello que este proyecto de investigación se enfocó en la relación entre el desempeño docente y la tasa de aprobación de la Carrera de Medicina en el periodo 2017 y en determinar causas que pudieran afectar la aprobación de estudiantes en los cursos.

El ejercicio de la docencia en la carrera de medicina cuenta con un profesorado con altas competencias profesionales en su campo 
disciplinar y en constante preparación en el área pedagógica promovida por la universidad o por intereses individuales.

Los resultados de la tasa de aprobación únicamente brindan un dato cuantitativo con pocas posibilidades de determinar las causas que lo determinan; mientras que la evaluación del desempeño docente brinda información cualitativa que permite conocer el papel docente en el desarrollo del curso.

\section{Marco teórico}

Cualquier tipo de evaluación es fundamental para mejorar un producto, proceso o el desempeño de quienes colaboran en él. La evaluación es la base para lograr personas competitivas en el mercado y el éxito de las operaciones.

La evaluación es la apreciación sistemática del valor que un individuo demuestra, por sus características personales y/o por su prestación, expresado periódicamente conforme a un preciso procedimiento aplicado a una o más personas y que conozcan al individuo y su trabajo. (Puchol, 2007, p. 259)

La evaluación del desempeño docente en el aula tiene como objetivo primordial proveer oportunidades de aprendizaje a todo el estudiantado según los objetivos propuestos para alcanzar los conocimientos y las habilidades descritas en el perfil académico-profesional.

El desempeño del personal docente está relacionado con el cumplimiento de determinadas obligaciones o actividades en el contexto curricular con el fin de responder a las exigencias académicas y sociales. "El currículum no se limita a un listado de asignaturas sino que se concreta en un documento y una práctica en el aula que los aprendizajes son significativos siguiendo una secuencia sistemática" (Molina, 2011, p. 22).

Los cuerpos docentes deben ser capaces de planificar y desarrollar las actividades programadas con el fin de dar respuesta a los requerimientos de la comunidad universitaria respetando las desigualdades y promoviendo el desarrollo de las capacidades individuales.

La carrera de Medicina responde a un plan de estudios conformado por 52 asignaturas a lo largo de doce cuatrimestres para un total de 184 créditos. El plan de estudios responde a un modelo por objetivos, 
con un enfoque teórico práctico que incorpora estrategias de aprendizajes y estrategias de evaluación en cada uno de los programas de asignaturas, según su naturaleza.

La evaluación del desempeño profesional del docente es un proceso sistemático de obtención de datos fiables, con el objetivo de comprobar y valorar el efecto educativo que produce en los alumnos el despliegue de sus capacidades pedagógicas, su emocionalidad, responsabilidad laboral y la naturaleza de sus relaciones interpersonales. (Posner, 1998, p. 216)

Aunado a lo anterior, Montenegro (2007) señala:

La evaluación del desempeño docente puede hacerse a raíz de cuatro enfoques establecidos: basados en el perfil del docente, basados en los resultados alcanzados en el curso, basados en lo que el docente hace en la práctica educativa en el aula y basado en la reflexión o análisis entre pares. (p. 68)

En la Carrera de Medicina el profesorado es evaluado cuatrimestralmente por sus estudiantes, con el fin de determinar específicamente su desempeño como docentes en el aula. La evaluación del desempeño mide aspectos administrativos y aspectos de caracteres metodológicos relacionados con el proceso de enseñanza y aprendizaje. En los aspectos administrativos se califica el cumplimiento de los horarios, la motivación, la asistencia, el cronograma establecido, la interacción con estudiantes y la preparación de las lecciones. Dentro de los aspectos metodológicos se evalúa el dominio de los contenidos, la capacidad pedagógica y las estrategias de evaluación utilizadas, entre otros aspectos.

La universidad desarrolla una cultura de evaluación entre su personal docente, administrativo y de apoyo como una forma de identificar insuficiencias para superarlas o fortalezas que puedan ser compartidas entre escuelas o facultades. La evaluación del desempeño, los méritos académicos y la experiencia docente son importantes para determinar la categoría del personal instructor, profesor adjunto, o catedrático en el escalafón docente de la institución. 
La cultura evaluativa es definida por Cariola (2003) como "productora de información objetiva que permite disminuir la resistencia de los docentes en los procesos de mejoramiento de la carrera" (p.112).

Según Prieto (2007), "los principales desafíos de la evaluación del desempeño es que contribuya de forma efectiva, al logro de objetivos relacionados con el desarrollo institucional de la universidad, la mejora de la enseñanza, el crecimiento personal y profesional de los docentes" (p. 165).

La dirección de la carrera ejerce un control eficaz sobre la ejecución del plan de estudios a través de un procedimiento que implica la evaluación de docentes. En la semana ocho se aplica la encuesta al estudiantado donde se evalúa la puntualidad, el dominio de la materia, justicia de la evaluación consignada en el programa y cumplimiento de los objetivos y contenidos del programa por la parte docente. En la semana 14 se aplica la encuesta de final del cuatrimestre o la evaluación del desempeño docente.

La evaluación del desempeño docente es aplicada por la Oficina de Calidad Académica de la Universidad, una vez por cuatrimestre, a todo el profesorado y es completada por la totalidad del alumnado matriculado en los cursos. La información es procesada de inmediato y los resultados son entregados a cada docente por el director o directora de carrera u otra autoridad académica, con el objetivo de que sirva de retroalimentación en el proceso educativo.

Al respecto, Cabra (2013) señala que "en la medida que la evaluación docente se transforme en una instancia altamente participativa en lo institucional, académico y estudiantil; el mejoramiento de la calidad de la educación es más factible" (p. 91).

Para la Universidad, la evaluación del personal docente es una manera de identificar las cualidades que conforman un buen profesorado y, a la vez, determinar las necesidades de capacitación para el diseño de acciones encaminadas a su desarrollo de competencias pedagógicas.

Desde el año 2007, la Universidad desarrolla un programa de desarrollo profesional con el fin de elevar las competencias pedagógicas de sus docentes. Este programa pretende dar respuesta tanto a las necesidades individuales del profesorado como a las de la propia institución, y constituye un esfuerzo por lograr un ambiente de interacción y comunicación constante, como apoyo al ejercicio de la profesión en el aula de clase. 
La evaluación del desempeño en la Universidad forma parte de una política de control y mejoramiento de las competencias del personal académico para asegurar la calidad de la labor docente en cada curso y definir o aplicar acciones correctivas de mejora en forma pronta y oportuna. Las evaluaciones del desempeño docente se llevan a cabo a nivel institucional desde el año 1999.

En la investigación se recurrió a la revisión de bibliografía que fundamentara el desarrollo de un marco teórico referido a la evaluación docente. También, se llevó a cabo una revisión de la normativa de la Universidad en cuanto a las funciones y deberes de sus docentes en el proceso educativo.

El Estatuto Orgánico de la Universidad, Artículo 46, describe las obligaciones de cada docente en los siguientes términos:

- Entregar el programa del curso a los estudiantes en la primera lección, con la especificación de los criterios de evaluación y sus respectivas ponderaciones. Las normas de evaluación, una vez hechas del conocimiento de los estudiantes, no podrán ser variadas por el profesor sin el consentimiento unánime del grupo.

- Preparar las lecciones, material didáctico, prácticos y visitas necesarias para el desarrollo del curso bajo su responsabilidad.

- Confeccionar y calificar tareas, pruebas de evaluación continuas, parciales, finales, extraordinarias, etc.; para supervisar el rendimiento académico de los alumnos. Entregar a los alumnos los exámenes calificados a más tardar quince días naturales después de efectuados.

La Oficina de Calidad Académica controla sistemáticamente lo relacionado con la evolución del alumnado matriculado. Al finalizar el cuatrimestre se obtiene la tasa de aprobación, cantidad de estudiantes que reprobaron y el porcentaje de estudiantes que abandonaron cada uno de los cursos de la carrera. En el año 2017 el comportamiento de la tasa de aprobación fue significativamente mayor que la tasa de reprobación y deserción estudiantiles. La tasa de mayor reprobación de estudiantes se presentó en determinados cuatrimestres del plan de estudios como puede apreciarse en las Tablas 1, 2 y 3 . 
Tabla 1

Distribución de docentes del I cuatrimestre 2017 con mayor tasa de reprobación, según su ubicación en el programa de estudios

\begin{tabular}{|c|c|}
\hline Cuatrimestre de estudio & Docentes con mayor tasa de reprobación \\
\hline 4 & 8 \\
\hline 5 & 5 \\
\hline 1 & 5 \\
\hline 8 & 4 \\
\hline
\end{tabular}

Nota: Información recolectada por la Oficina de Calidad Académica Universidad Federada de Costa Rica.

\section{Tabla 2}

Distribución de docentes con mayor tasa de reprobación según la ubicación del curso en el programa de estudios del II cuatrimestre 2017

\begin{tabular}{|c|c|}
\hline Cuatrimestre de estudio & Docentes con mayor tasa de reprobación \\
\hline 4 & 7 \\
\hline 3 & 6 \\
\hline 5 & 6 \\
\hline 2 & 5 \\
\hline
\end{tabular}

Nota: Información recolectada por la Oficina de Calidad Académica Universidad Federada de Costa Rica.

\section{Tabla 3}

Distribución de docentes con mayor tasa de reprobación, según la ubicación del curso en el programa de estudios del III cuatrimestre 2017

\begin{tabular}{|c|c|}
\hline Cuatrimestre de estudio & Docentes con mayor tasa de reprobación \\
\hline 4 & 11 \\
\hline 3 & 6 \\
\hline 5 & 5 \\
\hline 8 & 5 \\
\hline
\end{tabular}

Nota: Información recolectada por la Oficina de Calidad Académica Universidad Federada de Costa Rica. 


\section{Metodología}

El estudio tiene un enfoque cuantitativo, de corte transversal, con un alcance exploratorio-descriptivo; ya que el objetivo es identificar la posible relación entre el comportamiento de la tasa de aprobación de estudiantes de la Facultad de Medicina de los 3 cuatrimestres del periodo 2017 con los resultados de la evaluación del desempeño de docentes en el mismo periodo.

El alcance exploratorio, según Hernández, Fernández y Baptista (2014), tiene la siguiente finalidad:

Familiarizarnos con fenómenos relativamente desconocidos, obtener información sobre la posibilidad de llevar a cabo una investigación más completa respecto de un contexto particular, indagar nuevos problemas, identificar conceptos o variables promisorias, establecer prioridades para investigaciones futuras, o sugerir afirmaciones y postulados. (p. 91)

El comportamiento de las tasas de aprobación en contextos universitarios ha sido poco estudiado; así como la relación que puede tener el desempeño docente en el estudiantado. Por esta razón, se consideró pertinente realizar esta investigación para analizar el comportamiento de los índices de aprobación y contrastarlos con los resultados de la evaluación del desempeño docente, la cual es calificada por el estudiantado al final de cuatrimestre.

\section{Participantes}

Para este estudio se consideraron los cursos y docentes de la Facultad de Medicina que fueron calificados en el I, II y III cuatrimestre del periodo 2017 ( $\mathrm{N}=259)$. Se logró identificar la muestra de un 98.8 $\%(\mathrm{n}=256)$. Se consideró como criterio de inclusión a docentes que el estudiantado hubiera evaluado al final de cada cuatrimestre.

\section{Proceso de investigación}

En párrafos anteriores, se indicaron los criterios de inclusión que se utilizaron para seleccionar la muestra; esto permitió al equipo de investigación relacionar el resultado de la tasa de aprobación y de la evaluación del desempeño de docentes de la Facultad de Medicina. Lo 
anterior evidencia el alcance exploratorio de este estudio. Se considera oportuno indicar las categorías de la evaluación del desempeño que utiliza la Oficina de Calidad de la Universidad Federada de Costa Rica, San Judas Tadeo (Tabla 4):

Tabla 4

Distribución de nota evaluación docente según categoría

\begin{tabular}{|c|l|}
\hline $\begin{array}{c}\text { Nota de evaluación del } \\
\text { desempeño docente }\end{array}$ & \multicolumn{1}{|c|}{ Categoría } \\
\hline 0 a 68 & Deficiente desempeño \\
\hline 70 a 78 & Regular desempeño \\
\hline 80 a 88 & Buen desempeño \\
\hline 90 a 100 & Excelente desempeño \\
\hline
\end{tabular}

Nota: Información recolectada por la Oficina de Calidad Académica Universidad Federada de Costa Rica.

Como se indica en la tabla 4, docentes que obtienen una nota igual o mayor a 80 se encuentran entre las categorías "bueno" y "excelente"; por lo tanto, para este estudio el resultado de la evaluación docente que sea igual o mayor a 80 será considerado como "satisfactorio" y docentes que obtuvieron una nota menor a 80 en su desempeño será considerado como "no satisfactorio".

Acerca de la tasa de aprobación, se tomó como base la nota que requiere el estudiante para aprobar una materia; por lo tanto, la tasa de aprobación igual o mayor a 70 fue considerada como "adecuada" y los índices menores a 70 serán considerados como "no adecuados".

Para una mejor comprensión de los resultados de este estudio, el equipo investigador realizó el análisis a partir de la definición de variables e indicadores; los cuales se describirán a continuación:

\section{Relación simétrica}

Se da cuando se tiene el mismo valor (positivo o negativo) entre la evaluación docente y la tasa de aprobación. Ejemplo: satisfactorio desempeño docente y adecuada tasa de aprobación o insatisfactoria evaluación docente y una inadecuada tasa de aprobación.

La relación simétrica puede darse de dos maneras: 
Relación simétrica positiva: Se da cuando los resultados evidencian un satisfactorio desempeño docente y adecuada tasa de aprobación de estudiantes.

Relación simétrica negativa: Se da cuando los resultados reflejan insatisfactorio desempeño docente y una inadecuada tasa de aprobación de estudiantes.

\section{Relación asimétrica}

Se da cuando se tienen diferentes valores (positivo y negativo) entre la evaluación docente y la tasa de aprobación. Ejemplo: satisfactorio desempeño docente y una inadecuada tasa de aprobación o insatisfactoria evaluación del desempeño docente y adecuada tasa de aprobación de estudiantes.

La relación asimétrica puede darse de dos maneras:

Relación asimétrica positiva: se da cuando los resultados reflejan que hay insatisfactorio desempeño docente y adecuada tasa de aprobación de estudiantes.

Relación asimétrica negativa: se da cuando los resultados obtenidos evidencian que es satisfactoria la evaluación del desempeño docente y una inadecuada tasa de aprobación de estudiantes.

Aunado a lo anterior, se realizó una descripción de los ítems de la evaluación del desempeño docente en los tres cuatrimestres del periodo 2017, que fueron calificados por el estudiantado de forma negativa.

\section{Resultados y análisis}

\section{Relación entre variables}

A continuación se detallan los resultados del desempeño de la evaluación docente de la Facultad de Medicina, la cual es aplicada al final de los 3 cuatrimestres del periodo 2017 y estos se relacionaron con la tasa de aprobación de estudiantes. 
Como se muestra en la Figura 1, existe relación de 69 \% entre la tasa de aprobación y el desempeño de docente. También se identificó que en algunos cursos (31\%) no hay relación alguna entre los índices de aprobación de estudiantes con la nota del desempeño de docentes.

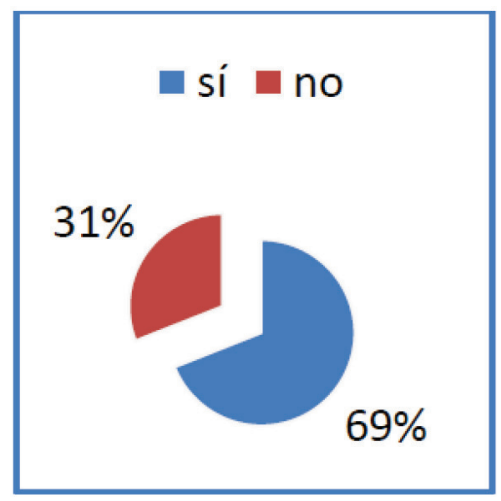

\section{Figura 1}

Relación entre la evaluación del desempeño docente y la tasa de aprobación de estudiantes de la Facultad de Medicina del I cuatrimestre, 2017

Nota: Datos suministrados por la Oficina de Calidad Académica de la Universidad Federada de Costa Rica San Judas Tadeo.

Como se indica en la Figura 2, los resultados del II cuatrimestre del periodo 2017 muestran que existe una relación de $57 \%$ entre la evaluación del desempeño docente y la tasa de aprobación de estudiantes de la Facultad de Medicina. Sin embargo, también se logró identificar que no hay relación (43\%) entre la tasa de aprobación de estudiantes con el desempeño docente.

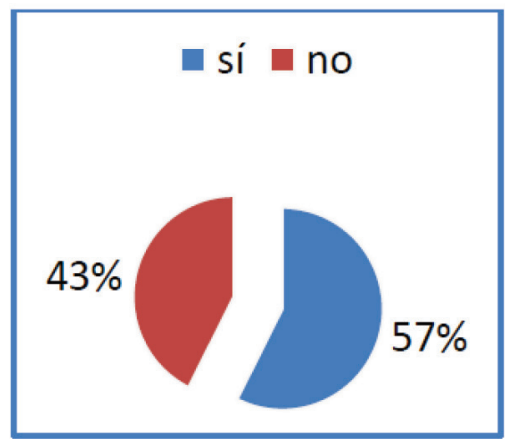

\section{Figura 2}

Relación entre la evaluación del desempeño docente y la tasa de aprobación de estudiantes de la Facultad de Medicina del II cuatrimestre, 2017

Nota: Datos suministrados por la Oficina de Calidad Académica de la Universidad Federada de Costa Rica San Judas Tadeo. 
Como se indica en la Figura 3, en el III cuatrimestre del periodo 2017 se presentó una relación de $52 \%$ entre evaluación del desempeño docente y la tasa de aprobación por parte de estudiantes de la facultad de medicina. También se identificó que en un $48 \%$ de los cursos no hay relación entre la evaluación del desempeño docente y la tasa de aprobación de estudiantes.

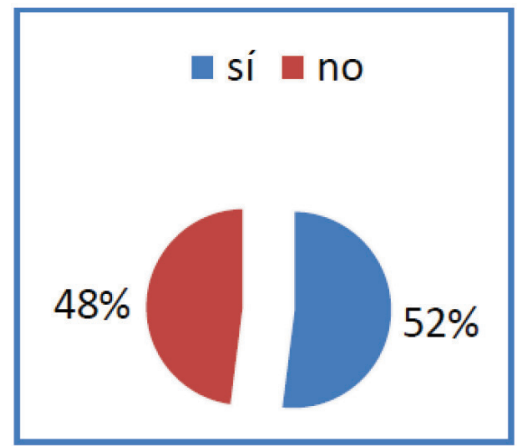

\section{Figura 3}

Relación entre la evaluación del desempeño docente y la tasa de aprobación de estudiantes de la Facultad de Medicina del III cuatrimestre, 2017

Nota: Datos suministrados por la Oficina de Calidad Académica de la Universidad Federada de Costa Rica San Judas Tadeo.

La Figura 4 refleja que en los 3 cuatrimestres se presentó una relación entre el desempeño docente y la tasa de aprobación de los estudiantes de la Facultad de Medicina; en promedio, la relación se presentó en el $59 \%$ de los cursos durante el periodo 2017. Es decir, que en la mayoría de los cursos se evidenció un desempeño docente satisfactorio (igual o mayor a 80) y una tasa de aprobación estudiantil adecuada (igual o mayor a 70); algunos de los resultados indicaron un desempeño docente insatisfactorio (menor a 80) y los índices de tasa de aprobación estudiantil no adecuada (menor a 70).

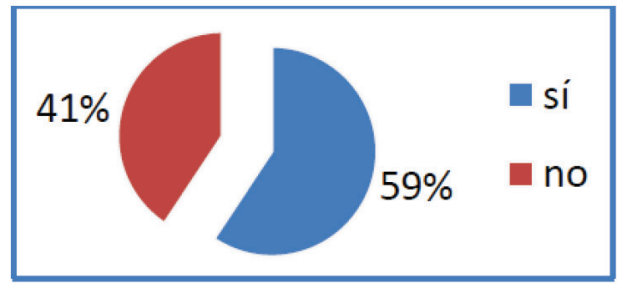

\section{Figura 4}

Relación entre la evaluación del desempeño docente y la tasa de aprobación de estudiantes de la Facultad de Medicina del periodo 2017

Nota: Datos suministrados por la Oficina de Calidad Académica de la Universidad Federada de Costa Rica San Judas Tadeo. 


\section{Tipo de relación simétrica entre variables}

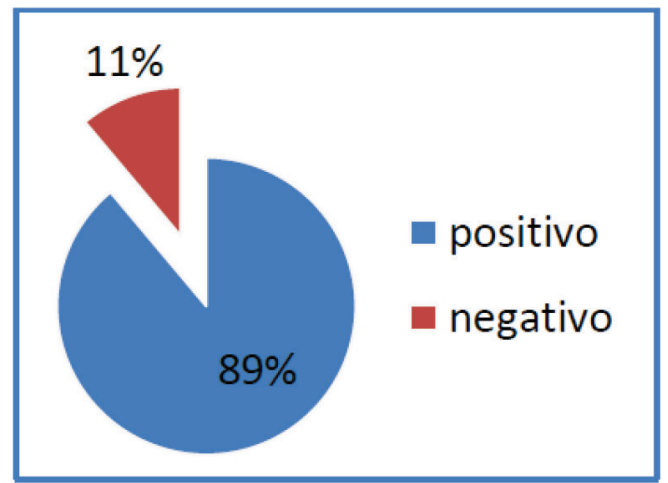

\section{Figura 5}

Tipo de relación simétrica

del I cuatrimestre 2017

Nota: Datos suministrados por la Oficina de Calidad Académica de la Universidad Federada de Costa Rica San Judas Tadeo.

En el I cuatrimestre del periodo 2017, del $69 \%$ de los cursos que tienen relación entre la evaluación del desempeño docente y la tasa de aprobación de estudiantes, el $92 \%$ fue positivo, es decir, los resultados indicaron que se dio un satisfactorio desempeño de docentes (de 80 en adelante) y una adecuada tasa de aprobación de estudiantes (de 70 en adelante), lo que significa que estudiantes que evaluaron bien el desempeño de sus docentes también obtuvieron una adecuada tasa de aprobación.

El $8 \%$ arrojó una relación negativa, es decir, insatisfactorio desempeño docente (de 79 o menos) y una tasa de aprobación no adecuada por parte de estudiantes (de 69 o menos).

Como se muestra en la Figura 6, en el II cuatrimestre del periodo 2017, del $57 \%$ de los cursos que tienen relación simétrica entre la evaluación del desempeño docente y la tasa de aprobación de estudiantes, la relación predominante es la positiva $(98 \%)$. 


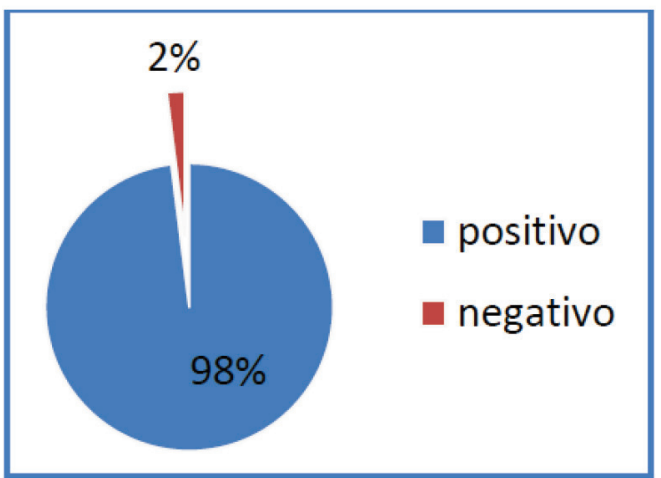

Figura 6

Tipo de relación simétrica

del II cuatrimestre 2017

Nota: Datos suministrados por la Oficina de Calidad Académica de la Universidad Federada de Costa Rica San Judas Tadeo.

La relación simétrica positiva quiere decir que el personal docente obtuvo una nota satisfactoria en su desempeño (de 80 en adelante) y la tasa de aprobación por parte de sus estudiantes fue adecuada (la tasa fue de 70 en adelante). Ello significa que el desempeño de docentes fue bien evaluado y el estudiantado obtuvo una adecuada tasa de aprobación. En el mismo periodo la relación simétrica negativa fue del $2 \%$, es decir, el desempeño de docentes fue insatisfactorio (menor a 80) y la tasa de aprobación de estudiantes fue inadecuada (menor a 70).

Como se describe en la Figura 7, del $52 \%$ de los cursos del III cuatrimestre 2017 que tienen relación entre la evaluación del desempeño docente y la tasa de aprobación de estudiantes, la relación predominante es la positiva $(95 \%)$.

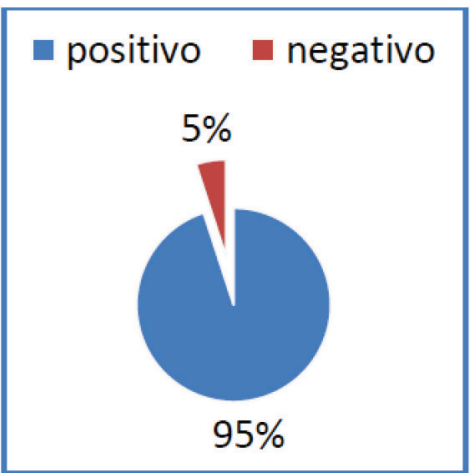

Figura 7

Tipo de relación simétrica del III cuatrimestre 2017

Nota: Datos suministrados por la Oficina de Calidad Académica de la Universidad Federada de Costa Rica San Judas Tadeo. 
La relación simétrica positiva significa que el personal docente obtuvo una nota satisfactoria en la evaluación de su desempeño (igual o mayor a 80) y la tasa de aprobación por parte del estudiantado de medicina fue adecuada (igual o mayor a 70). También se identificó que la relación simétrica negativa es mínima (5\%). La relación simétrica negativa significa que los resultados de la evaluación del desempeño docente no fueron satisfactorios, y la tasa de aprobación de estudiantes de medicina tampoco fue la adecuada.

La figura 8 señala que en los 3 cuatrimestres del periodo 2017 prevaleció una relación simétrica positiva $(95 \%)$ entre el desempeño docente y la tasa de aprobación de estudiantes de la Facultad de Medicina. Estos datos evidencian que en la mayoría de los cursos el desempeño de docentes fue satisfactorio (igual o mayor a 80 ) y una tasa de aprobación estudiantil adecuada (igual o mayor a 70). Mientras que en la minoría de los cursos ( $5 \%$ ) los resultados indicaron un desempeño docente insatisfactorio (menor a 80 ) y la tasa de aprobación estudiantil fue no adecuada (menor a 70).

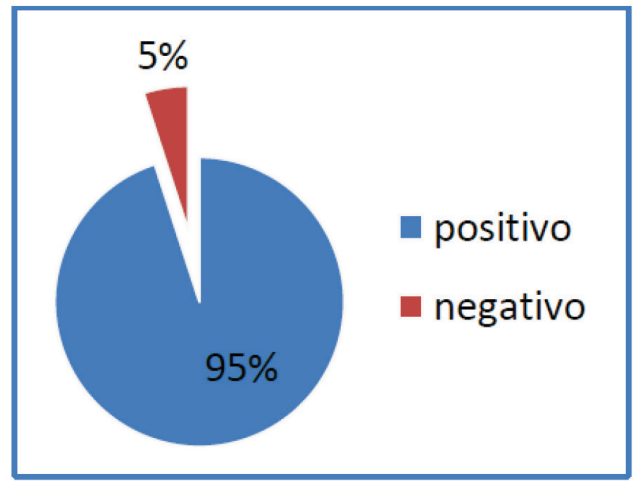

Figura 8

Resumen del tipo de relación simétrica del periodo 2017

Nota: Datos suministrados por la Oficina de Calidad Académica de la Universidad Federada de Costa Rica San Judas Tadeo. 


\section{Tipo de relación asimétrica entre variables}

La figura 9 señala que en el $31 \%$ de los cursos con relación asimétrica, la relación que prevalece es la negativa ( $75 \%$ ), lo que significa que el desempeño de docentes fue bien evaluado por sus estudiantes (obtuvieron una nota igual o mayor a 80), pero la tasa de aprobación por parte de estudiantes no fue adecuada (menor a 70). Por otro lado, en la relación asimétrica positiva ( $25 \%$ ) significa que los resultados de la evaluación del desempeño docente fueron insatisfactorios (obtuvieron una nota menor a 80), pero la tasa de aprobación por parte de estudiantes fue adecuada (igual a mayor a 70).

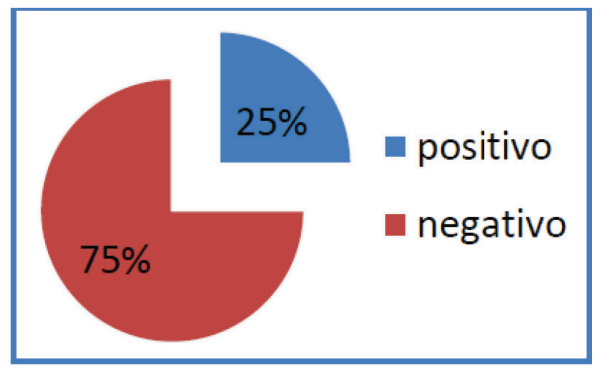

Figura 9

Tipo de relación asimétrica del I cuatrimestre 2017

Nota: Datos suministrados por la Oficina de Calidad Académica de la Universidad Federada de Costa Rica San Judas Tadeo.

La Figura 10 indica que del $43 \%$ en los cursos del II cuatrimestre del periodo 2017, la relación que predominó fue la asimétrica negativa ( $82 \%$ ); es decir, el desempeño docente fue bien evaluado (igual o mayor a 80 ), pero la tasa de aprobación no fue adecuada (menor a 70). La relación asimétrica positiva significa que el $18 \%$ de docentes fue mal evaluado en su desempeño (obtuvo una nota menor a 80 ), pero la tasa de aprobación de estudiantes fue adecuada (la tasa fue igual o mayor a 70).

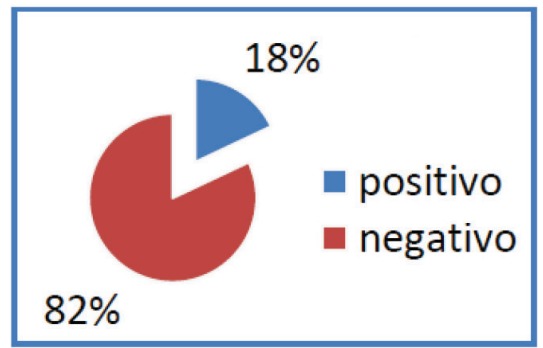

Figura 10

Tipo de relación asimétrica del II cuatrimestre 2017

Nota: Datos suministrados por la Oficina de Calidad Académica de la Universidad Federada de Costa Rica San Judas Tadeo. 
En la figura 11, se observa que del $48 \%$ en los cursos que se impartieron en el III cuatrimestre la relación que prevaleció fue la negativa (90 \%) entre los resultados de la evaluación del desempeño docente y la tasa de aprobación de estudiantes de la Facultad de Medicina. La relación asimétrica negativa significa que el desempeño docente fue bien evaluado por el estudiantado (igual o mayor a 80); sin embargo, su tasa de aprobación no fue adecuada (menor a 70). También se identificó que la relación asimétrica positiva fue mínima (10\%); es decir, el resultado de la evaluación del desempeño docente fue insatisfactoria (menos de 80) y la tasa de aprobación de estudiantes fue adecuada (igual o mayor a 70).

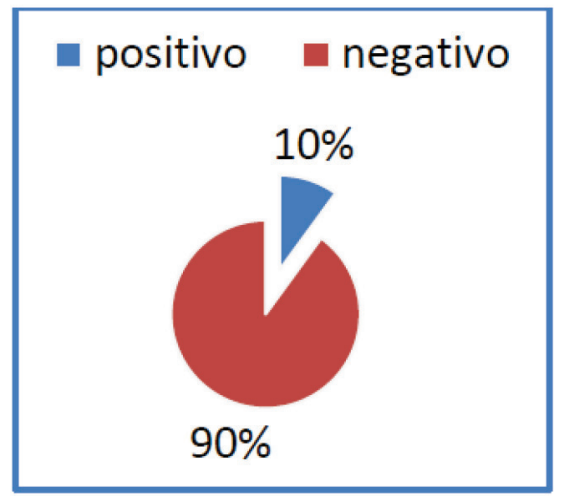

Figura 11

Tipo de relación asimétrica del III cuatrimestre 2017

Nota: Datos suministrados por la Oficina de Calidad Académica de la Universidad Federada de Costa Rica San Judas Tadeo.

La Figura 12 señala que en los 3 cuatrimestres del periodo 2017 prevaleció una relación asimétrica negativa ( $82 \%$ ) entre el desempeño docente y la tasa de aprobación de estudiantes de la Facultad de Medicina. Estos datos evidencian que en la mayoría de los cursos el desempeño de docentes fue satisfactorio (igual o mayor a 80) mientras que la tasa de aprobación estudiantil no fue adecuada (menor a 70).

En la minoría de los cursos (5\%) los resultados indicaron un desempeño docente insatisfactorio (menor a 80) y la tasa de aprobación estudiantil fue adecuada (igual o mayor a 70). 
Relación entre la tasa de aprobación de estudiantes de la Facultad de Medicina y la evaluación del desempeño docente del periodo 2017

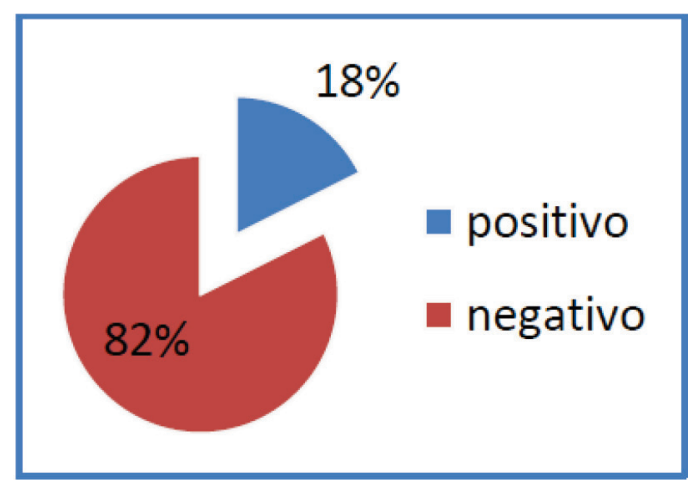

Figura 12

Tipo de relación asimétrica del periodo 2017

Nota: Datos suministrados por la Oficina de Calidad Académica de la Universidad Federada de Costa Rica San Judas Tadeo.

\section{Ítems que fueron mal evaluados por el estudiantado en el desempe- ño docente de los cursos con relación simétrica}

En el I cuatrimestre del 2017, en los cursos en que se identificó una relación simétrica negativa, los ítems de la evaluación del desempeño que fueron mal evaluados por el estudiantado son los siguientes:

Tabla 5

Ítems mal evaluados durante el I cuatrimestre 2017

\begin{tabular}{|c|c|c|}
\hline Ítem & Descripción & Porcentaje \\
\hline 1 & $\begin{array}{l}\text { Cumple el horario de inicio y finalización de la } \\
\text { clase }\end{array}$ & $43 \%$ \\
\hline 6 & $\begin{array}{l}\text { Cita ejemplos, explica con claridad los conceptos } \\
\text { y teorías de cada tema }\end{array}$ & $39.2 \%$ \\
\hline 7 & $\begin{array}{l}\text { Las clases están bien preparadas, organizadas y } \\
\text { estructuradas }\end{array}$ & $41.2 \%$ \\
\hline 8 & $\begin{array}{l}\text { Motiva y pregunta a todos para verificar } \\
\text { conocimientos impartidos }\end{array}$ & $44 \%$ \\
\hline 10 & Colabora con estudiantes que tienen dificultades & $49.2 \%$ \\
\hline 11 & Logra que estudiantes se interesen por la materia & $45 \%$ \\
\hline 15 & $\begin{array}{l}\text { Los recursos didácticos utilizados ayudan a } \\
\text { comprender mejor los contenidos de la asignatura }\end{array}$ & $25.4 \%$ \\
\hline
\end{tabular}

Nota: Datos suministrados por la Oficina Calidad Académica Universidad Federada de Costa Rica San Judas Tadeo (2018). 
En el II cuatrimestre del 2017, en los cursos en que se identificó una relación simétrica negativa, los ítems que fueron mal evaluados por estudiantes fueron:

\section{Tabla 6}

Ítems mal evaluados durante el II cuatrimestre 2017

\begin{tabular}{|c|l|c|}
\hline Ítem & \multicolumn{1}{|c|}{ Descripción } & Porcentaje \\
\hline 5 & Evidencia un dominio de la materia & $100 \%$ \\
\hline 6 & $\begin{array}{l}\text { Cita ejemplos, explica con claridad los conceptos } \\
\text { y teorías de cada tema }\end{array}$ & $89 \%$ \\
\hline 7 & $\begin{array}{l}\text { Las clases están bien preparadas, organizadas y } \\
\text { estructuradas }\end{array}$ & $100 \%$ \\
\hline 8 & $\begin{array}{l}\text { Motiva y pregunta a todo el grupo para verificar } \\
\text { conocimientos impartidos }\end{array}$ & $78 \%$ \\
\hline 10 & Colabora con estudiantes que tienen dificultades & $44 \%$ \\
\hline 11 & Logra que estudiantes se interesen por la materia & $89 \%$ \\
\hline 12 & $\begin{array}{l}\text { Aplica las evaluaciones según lo planificado en } \\
\text { el programa }\end{array}$ & $44 \%$ \\
\hline 15 & $\begin{array}{l}\text { Los recursos didácticos utilizados ayudan a } \\
\text { comprender mejor los contenidos de la asignatura }\end{array}$ & $89 \%$ \\
\hline
\end{tabular}

Nota: Datos suministrados por la Oficina Calidad Académica Universidad Federada de Costa Rica San Judas Tadeo (2018)

En el III cuatrimestre del 2017, en los cursos en que se identificó una relación simétrica negativa, los ítems que fueron mal evaluados por estudiantes fueron: 
Relación entre la tasa de aprobación de estudiantes de la Facultad de Medicina y la evaluación del desempeño docente del periodo 2017

\section{Tabla 7}

Ítems mal evaluados durante el II cuatrimestre 2017

\begin{tabular}{|c|l|c|}
\hline Ítem & \multicolumn{1}{|c|}{ Descripción } & Porcentaje \\
\hline 7 & $\begin{array}{l}\text { Las clases están bien preparadas, organizadas y } \\
\text { estructuradas }\end{array}$ & $45.5 \%$ \\
\hline 8 & $\begin{array}{l}\text { Motiva y pregunta a todo el grupo para verificar } \\
\text { conocimientos impartidos }\end{array}$ & $58.5 \%$ \\
\hline 9 & Muestra respeto y exigencia con estudiantes & $51.5 \%$ \\
\hline 10 & Colabora con estudiantes que tienen dificultades & $58.5 \%$ \\
\hline 13 & $\begin{array}{l}\text { Acepta revisar la calificación en caso de } \\
\text { reclamación y posibles errores }\end{array}$ & $48.5 \%$ \\
\hline 14 & $\begin{array}{l}\text { Entrega las notas de las evaluaciones en el plazo } \\
\text { establecido (14 días) }\end{array}$ & $41.5 \%$ \\
\hline 15 & $\begin{array}{l}\text { Los recursos didácticos utilizados ayudan a } \\
\text { comprender mejor los contenidos de la asignatura }\end{array}$ & $58.5 \%$ \\
\hline
\end{tabular}

Nota: Datos suministrados por la Oficina Calidad Académica Universidad Federada de Costa Rica San Judas Tadeo (2018).

En la Figura 13 se visualizan los ítems mal evaluados por los estudiantes en los 3 cuatrimestres del periodo 2017

\section{Figura 13}

Resumen de items que fueron mal evaluados por estudiantes en los cursos en que se identificó una relación simétrica del periodo 2017

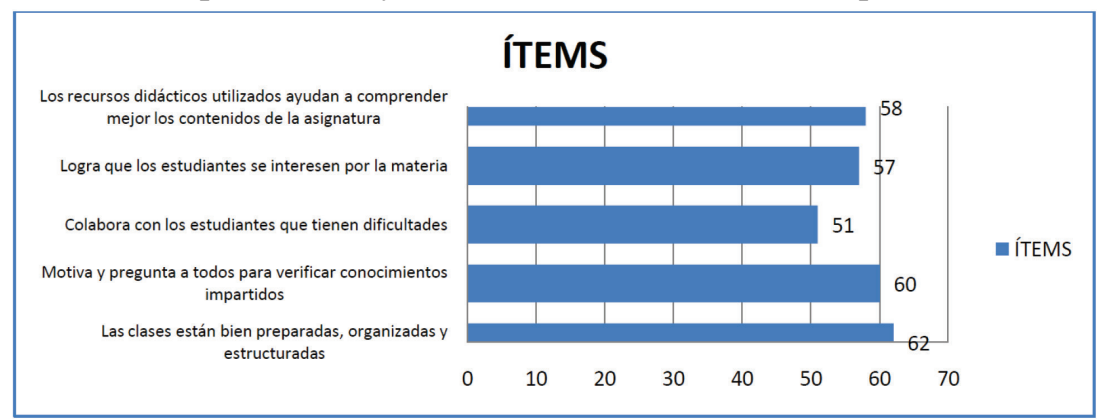

Nota: Datos suministrados por la Oficina de Calidad Académica de la Universidad Federada de Costa Rica San Judas Tadeo. 


\section{Ítems que fueron mal evaluados por estudiantes en el desempeño docente de los cursos con relación asimétrica}

En el I cuatrimestre del 2017, en los cursos en que se identificó una relación asimétrica negativa, los ítems que fueron mal evaluados por estudiantes fueron:

Tabla 8

Ítems mal evaluados durante el I cuatrimestre 2017

\begin{tabular}{|c|l|c|}
\hline Ítem & \multicolumn{1}{|c|}{ Descripción } & Porcentaje \\
\hline 1 & $\begin{array}{l}\text { Cumple el horario de inicio y finalización de la } \\
\text { clase }\end{array}$ & $7.9 \%$ \\
\hline 6 & $\begin{array}{l}\text { Cita ejemplos, explica con claridad los conceptos } \\
\text { y teorías de cada tema }\end{array}$ & $9.9 \%$ \\
\hline 7 & $\begin{array}{l}\text { Las clases están bien preparadas, organizadas y } \\
\text { estructuradas }\end{array}$ & $10.4 \%$ \\
\hline 8 & $\begin{array}{l}\text { Motiva y pregunta a todo el grupo para verificar } \\
\text { conocimientos impartidos }\end{array}$ & $7.8 \%$ \\
\hline 10 & Colabora con estudiantes que tienen dificultades & $9.4 \%$ \\
\hline 11 & Logra que estudiantes se interesen por la materia & $9.1 \%$ \\
\hline 15 & $\begin{array}{l}\text { Los recursos didácticos utilizados ayudan a } \\
\text { comprender mejor los contenidos de la asignatura }\end{array}$ & $13.3 \%$ \\
\hline
\end{tabular}

Nota: Datos suministrados por la Oficina Calidad Académica Universidad Federada de Costa Rica San Judas Tadeo (2018).

En el II cuatrimestre del 2017, en los cursos en que se identificó una relación asimétrica negativa, los ítems que fueron mal evaluados por estudiantes fueron: 
Tabla 9

Ítems mal evaluados durante el II cuatrimestre 2017

\begin{tabular}{|c|l|c|}
\hline Ítem & \multicolumn{1}{|c|}{ Descripción } & Porcentaje \\
\hline 6 & $\begin{array}{l}\text { Cita ejemplos, explica con claridad los conceptos } \\
\text { y teorías de cada tema }\end{array}$ & $18.2 \%$ \\
\hline 7 & $\begin{array}{l}\text { Las clases están bien preparadas, organizadas y } \\
\text { estructuradas }\end{array}$ & $19.8 \%$ \\
\hline 8 & $\begin{array}{l}\text { Motiva y pregunta a todo el grupo para verificar } \\
\text { conocimientos impartidos }\end{array}$ & $28.4 \%$ \\
\hline 10 & Colabora con estudiantes que tienen dificultades & $21.6 \%$ \\
\hline 11 & Logra que estudiantes se interesen por la materia & $24.2 \%$ \\
\hline 15 & $\begin{array}{l}\text { Los recursos didácticos utilizados ayudan a } \\
\text { comprender mejor los contenidos de la asignatura }\end{array}$ & $24.8 \%$ \\
\hline
\end{tabular}

Nota: Datos suministrados por la Oficina Calidad Académica Universidad Federada de Costa Rica San Judas Tadeo (2018).

En el III cuatrimestre del 2017, en los cursos en que se identificó una relación asimétrica negativa, los ítems que fueron mal evaluados por estudiantes fueron:

\section{Tabla 10}

Ítems mal evaluados durante el III cuatrimestre 2017

\begin{tabular}{|c|l|c|}
\hline Ítem & \multicolumn{1}{|c|}{ Descripción } & Porcentaje \\
\hline 7 & $\begin{array}{l}\text { Las clases están bien preparadas, organizadas y } \\
\text { estructuradas }\end{array}$ & $24.9 \%$ \\
\hline 8 & $\begin{array}{l}\text { Motiva y pregunta a todo el grupo para verificar } \\
\text { conocimientos impartidos }\end{array}$ & $13.4 \%$ \\
\hline 9 & Muestra respeto y exigencia con estudiantes & $9.9 \%$ \\
\hline 10 & Colabora con estudiantes que tienen dificultades & $15.9 \%$ \\
\hline 11 & Logra que estudiantes se interesen por la materia & $15.3 \%$ \\
\hline 15 & $\begin{array}{l}\text { Los recursos didácticos utilizados ayudan a } \\
\text { comprender mejor los contenidos de la asignatura }\end{array}$ & $18.9 \%$ \\
\hline
\end{tabular}

Nota: Datos suministrados por la Oficina Calidad Académica Universidad Federada de Costa Rica San Judas Tadeo (2018). 


\section{Figura 14}

Resumen de items que fueron mal evaluados por estudiantes en los cursos en que se identificó una relación asimétrica del periodo 2017

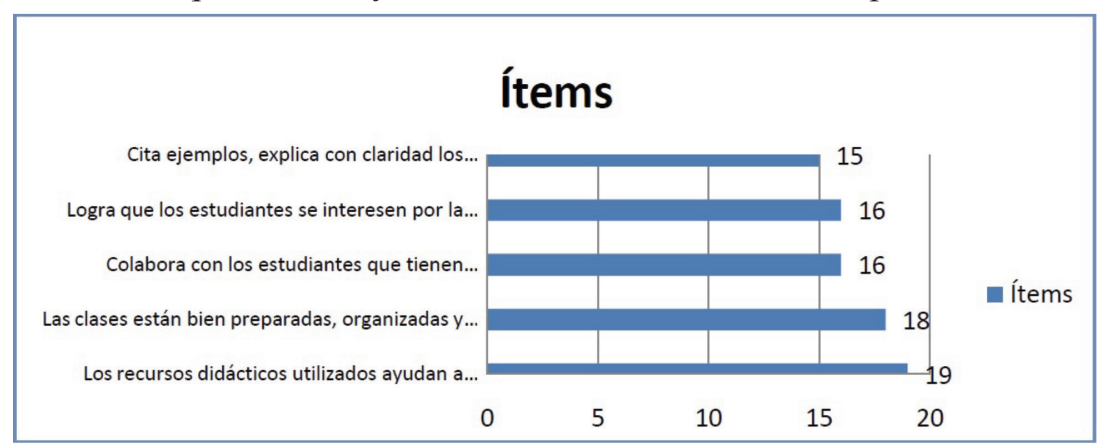

Nota: Datos suministrados por la Oficina de Calidad Académica de la Universidad Federada de Costa Rica San Judas Tadeo.

\section{Conclusiones}

Los resultados demuestran que existe una relación entre la evaluación del desempeño docente y la tasa de aprobación estudiantil de la Facultad de Medicina en los tres cuatrimestres del periodo 2017. En el $59 \%$ de los cursos se presentó una relación entre las variables analizadas, lo que significa que hubo una coincidencia, sea positiva o negativa, entre la evaluación del desempeño docente y la tasa de aprobación estudiantil.

El tipo de relación simétrica entre la evaluación del desempeño docente y la tasa de aprobación estudiantil que predominó en los 3 cuatrimestres fue la positiva (95\%); es decir, que el desempeño docente fue evaluado satisfactoriamente y la tasa de aprobación fue adecuada según los parámetros establecidos por la Universidad Federada de Costa Rica.

Por otro lado, el tipo de relación asimétrica entre la evaluación del desempeño docente y la tasa de aprobación estudiantil que predominó en los 3 cuatrimestres fue la negativa ( $82 \%$ ); es decir, que el desempeño docente fue evaluado satisfactoriamente y la tasa de aprobación fue inadecuada según los parámetros establecidos.

En los cursos donde se identificó la relación simétrica negativa, los ítems de las evaluaciones del desempeño docente que fueron mal evaluados por el estudiantado durante el periodo 2017 están relacionados con el 
cumplimiento del horario, la preparación y organización de las clases, la motivación de estudiantes, la utilización de los recursos didácticos utilizados, la colaboración con estudiantes en el proceso de aprendizaje.

En los cursos con una relación asimétrica negativa, los ítems que fueron mal evaluados por estudiantes en los últimos 3 cuatrimestres del periodo 2017 se relacionan con la mala utilización de los recursos didácticos para comprender mejor los contenidos de la asignatura, la organización y estructuración de las lecciones, la colaboración con estudiantes con dificultades, despertar el interés por los contenidos y en la forma de explicar conceptos y teorías.

La investigación arroja resultados de interés relacionados con el desempeño del profesorado en el aula. Se evidencia que la adecuada formación y la experiencia profesional de este grupo docente le permite un dominio de los contenidos que imparte y que debe mejorar algunos aspectos de índole actitudinal.

Aspectos metodológicos en la organización de las lecciones y la utilización apropiada de los recursos didácticos por el personal docente se ven claramente relacionados con la tasa de aprobación estudiantil.

Los resultados obtenidos serán tomados en cuenta en el Plan de Desarrollo de la Institución, con el objetivo de seguir perfeccionando la labor educativa docente, de manera que garantice mejores oportunidades de aprendizajes al estudiantado en su formación profesional en el área de la salud.

\section{Referencias}

Cabra, F. (2013). La evaluación y el enfoque de competencias: Tensiones, limitaciones y oportunidades. Colombia: Universidad EAN.

Cariola, L. (2003). Veinte años de políticas de educación en Chile. París: UNESCO.

Molina, G. B. (2011). Introducción al currículum. San José: EUNED.

Hernández, R., Fernández, C. y Baptista, P. (2014). Metodología de la investigación ( $6^{\text {ta }}$ ed.). México: Mc Graw-Hill.

Montenegro, I. (2007). Evaluación del desempeño docente. Fundamentos, modelos e instrumentos. Colombia: Magisterio.

Posner, G. (1998). Análisis del currículum. Colombia: McGrawHill.

Prieto, 1. (2007). Autoeficacia del profesor universitario. Eficacia y práctica docente. Madrid: Narcea. 
Puchol, L. (2007). Dirección y gestión de recursos humanos. Madrid: ESIC.

Universidad Federada de Costa Rica. (1994). Estatuto Orgánico. San José: Autor. 